\title{
Fracture of J-tipped guidewire during central venous catheterization and its successful removal under fluoroscopic guidance -A case report-
}

\author{
Sun Kyung Park, In-Kyong Yi, Jae-Ho Lee, Dae-Hee Kim, and Sook Young Lee \\ Department of Anesthesiology and Pain Medicine, Ajou University School of Medicine, Suwon, Korea
}

Central venous catheterization by Seldinger's technique uses a guidewire which may cause complications such as kinking, knotting and fracture. Fractured guidewire may lead to severe outcomes such as embolization, and removal of it may also cause problems such as vessel damage. We experienced a case of right internal jugular venous catheterization complicated by guidewire fracture entrapped in the central venous catheter, and its successful removal under fluoroscopic guidance using snare-loop. The patient recovered without any complications. When resistance is felt during insertion or withdrawal of the guidewire, force should not be applied to the guidewire and care should also be exercised when passing the tissue dilator over the guidewire. Clinicians should be aware of this rare complication and snare-loop technique could be considered as one of the methods for removal of the fractured guidewire. (Korean J Anesthesiol 2012; 63: 457-460)

Key Words: Central venous catheterization, Fracture, Guide wire.

Central venous catheters (CVCs) are used for the administration of drugs, hemodialysis/hemofiltration, hemodynamic monitoring and transvenous cardiac pacing. Large bore CVCs are used for the rapid infusion of resuscitation fluids [1]. Central venous catheterization is commonly performed by the Seldinger technique, which mandates the use of a guidewire $[2,3]$. Guidewire-associated complications include kinking, knotting, looping, breakage, and fracture [4-11]. Broken or fractured fragments of guidewire can lead to severe outcomes such as embolization or even cardiac arrest $[10,11]$. Removal of guidewire fragments may be performed using a snareloop catheter through the femoral vein $[12,13]$. There have only been a few rare reports of fracture and entrapment of CVC guidewires. We report a case of fractured guidewire that was entrapped in the CVC and its successful removal under fluoroscopic guidance using a snare- loop catheter.

Received: September 27, 2011. Revised: November 30, 2011. Accepted: December 15, 2011.

Corresponding author: Sook Young Lee, M.D., Ph.D., Department of Anesthesiology and Pain Medicine, Ajou University School of Medicine, San 5, Wonchun-dong, Youngtong-gu, Suwon 443-721, Korea. Tel: 82-31-219-4658, Fax: 82-31-219-5579, E-mail: anesylee@ajou.ac.kr (c) This is an open-access article distributed under the terms of the Creative Commons Attribution Non-Commercial License (http:// creativecommons.org/licenses/by-nc/3.0/), which permits unrestricted non-commercial use, distribution, and reproduction in any medium, provided the original work is properly cited. 


\section{Case Report}

A 63 year-old female patient was admitted to our hospital for laparoscopic splenectomy. Her diagnosis was ovarian cancer with splenic metastasis and there were no specific findings in the laboratory data and physical examination. At admission, blood pressure and heart rate were 100/ $63 \mathrm{mmHg}$ and 74 beats/ min, respectively. Anesthesia was induced with thiopental sodium (4-5 mg/kg) and was maintained with sevoflurane $2-2.5$ vol\%. The patients were given rocuronium (40 $\mathrm{mg}$ ) to facilitate tracheal intubation. Ventilation was mechanically controlled with $\mathrm{O}_{2}$ /air mixture (fractional inspired oxygen $\left[\mathrm{F}_{\mathrm{iO} 2}\right]$ $=0.5$ ) and adjusted to keep an end-tidal concentration of $\mathrm{CO}_{2}$ between 35 and $40 \mathrm{mmHg}$ throughout the operation.

After the induction of anesthesia, central venous catheterization through the right internal jugular vein was attempted with two $14 \mathrm{G}$ lumen indwelling catheter sets (Spectrum ${ }^{\circledR}$ Central venous Catheter Set, Cook Medical, USA). The enclosed introducer steel needle was used for puncture of the right internal jugular vein and free aspiration of blood was confirmed. The first attempt to insert the J-shaped guidewire failed with resistance. While lefting the introducer needle and guidewire, a more experienced clinician tried a second attempt and the guidewire passed through the venipuncture needle with resistance at the final step. The guidewire advanced approximately $19 \mathrm{~cm}$ beyond the needle tip. The tissue dilator was inserted over the guidewire without any problems. After removing the dilator, a double lumen catheter was inserted over the guidewire. More resistance was felt while attempting to withdraw the guidewire, so the guidewire was pulled out with force. The removed guidewire was observed to be damaged. The core wire was cut at the middle of the guidewire, and its surrounding cover coils detached and extended from the core wire (Fig. 1). It was not clear whether the J-shaped end was lost or not because the outer cover coil was extended long (Fig.

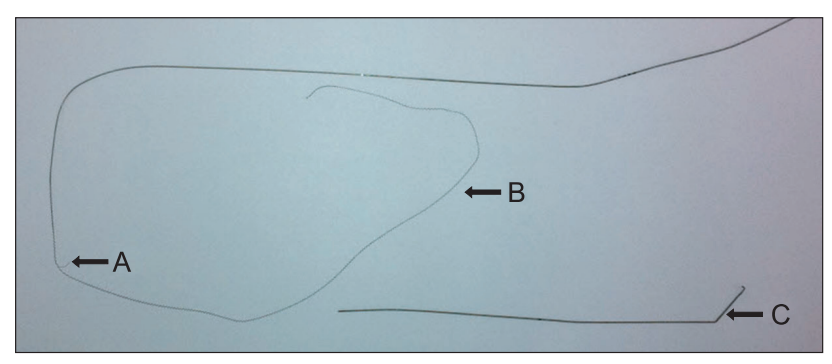

Fig. 1. Image of the fractured guidewire. (A) The cut end of the core wire from the proximal part of the fractured guidewire. (B) The detached and extended outer cover coil of the proximal part of the fractured guidewire. (C) The retrieved part of the guidewire $(19 \mathrm{~cm})$ in which the core wire and outer cover coils are fractured together without detaching the outer coil.
1). To confirm, chest $\mathrm{x}$-ray was taken, which revealed that the guidewire including the J-shaped tip was entrapped in the CVC (Fig. 2). We suspected fracture of the guidewire and a radiologist was called for correct diagnosis as well as to remove the guidewire. To remove the fractured fragment, a snareloop catheter 6 Fr. (Multi-Snare ${ }^{\circledR}$ set $30 \mathrm{~mm}$, pfm-prodokte für die Medizin AG, Köln, Germany) with a loop diameter of 30 $\mathrm{mm}$ was introduced through the femoral vein with the help of a fluoroscopic intensifier (Series 9800, GE OEC Medical Systems Inc., Cleveland, USA). The catheter passed into the superior vena cava adjacent to the J-shaped tip of the fractured guidewire. The snare was opened and slipped over the tip. The snare was tightened and the tip of the guidewire was withdrawn without difficulty. The removed fractured guidewire revealed a $19 \mathrm{~cm}$ length without detaching the outer cover coil. The guidewire curved from $3 \mathrm{~cm}$ proximal to the J-shaped tip by snare loop during removal of the guidewire. The total anesthesia and operation time was $295 \mathrm{~min}$ and $195 \mathrm{~min}$, respectively. The total time from the start of anesthesia induction to the removal of fragmented guidewire was $60 \mathrm{~min}$. Postoperatively, the patient was transferred to the general ward. The patient was discharged at postoperative days 9 without any complication associated with CVC.

\section{Discussion}

We report a case of fractured J-tipped guidewire being entrapped in the CVC during internal jugular venous catheterization and its successful removal with snare-loop catheter under fluoroscopic guidance.

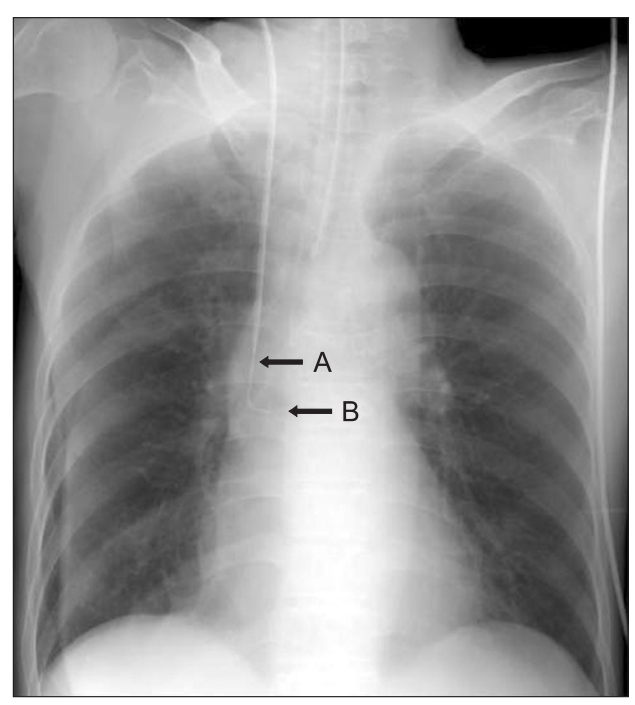

Fig. 2. Chest $x$-ray showing the fractured guidewire entrapped in the central venous catheter (CVC) located in the superior vena cava. (A) End of CVC. (B) End of fractured guidewire entrapped in the CVC. 
Central venous catheterization is associated with numerous complications including mechanical, infectious or thrombotic [12]. The reported rate of mechanical complications such as arterial puncture, air embolism, nerve injury and hemo- or pneumothorax is between $5 \%$ and $19 \%[14,15]$. Central venous catheterization is usually carried out by Seldinger's technique using guidewire which may cause complications such as failure to pass, loss in the vessel, kinking, knotting, breakage and fracture [4-11].

Safe use of guidewires for central venous access requires care in handling and understanding of the physical characteristics $[6,8]$. The guidewire consists of an inner single filament core wire and a surrounding coiled wire cover. Apart from the two ends of the guidewire, there is no further point of attachment between the core and the outer wire. Hence, any damage to the guidewire on its stem may lead to unwinding of the whole outer spiral. In our case, the outer coiled wire of the proximal part of the fractured guidewire was unwound. The J-shaped end results from rounding and flattening of the core which causes structural weakness and may lead to potential breakage $[6,8]$. In our case, the J-shaped end was damaged $3 \mathrm{~cm}$ distal from the tip of the end. However, the etiology of this damage may be the force applied to this part while removing the guidewire with the snare-loop. This could be evidenced by the initial chest radiograph showing an intact J-shaped end of the guidewire which was entrapped in the CVC (Fig. 2).

The removed guidewire (19 $\mathrm{cm}$ length) including the $\mathrm{J}$ - shaped end was fractured without the outer coil detachment. The proximal part of the fractured guidewire showed a cut core wire with a detached and stretched outer coil from the cut end (Fig. 1). The etiology of this complication could be described as follows. During guidewire insertion, we felt resistance mainly at the final step when the guidewire reached about 19 $\mathrm{cm}$ from the puncture needle tip (20 cm from the skin). At this time, initial damage to the guidewire due to the bevel of the accompanying puncture needle could have occurred. Although no problem was encountered while inserting the tissue dilator, secondary damage could be added to the guidewire since the initial damage had already occurred. During the insertion of the catheter with subsequent withdrawal of the guidewire, we also felt more resistance. However, we pulled out the guidewire with force. At this time, the guidewire may have been cut completely and the distal part of the fractured guidewire remained in the CVC. Further pulling of the guidewire may have caused the uncoiling of the outer cover coil of the proximal part of the guidewire. Monaca et al. [8] reported that if breakage occurred only in the inner part of the wire, it could exclude the effect of the needle. In our case, the inner wire and outer covering coil was fractured together and the damage to the guidewire could be from the puncture needle. Tissue dilators could cause

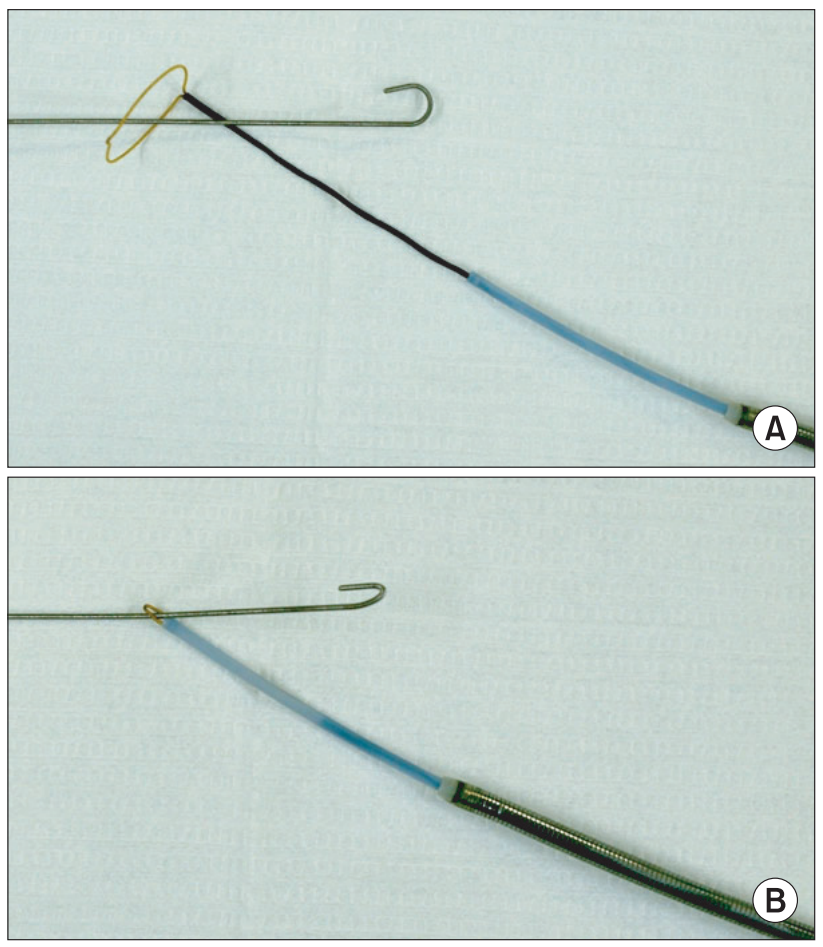

Fig. 3. Figure showing the J-tipped guidewire and snare-loop catheter. (A) Snare-loop slipped over the guidewire. (B) Tightened guidewire with snare-loop before being withdrawn.

complications to the guidewire [6]. In our case, there was no problem during insertion of the dilator. However, there may be damage to the guidewire because the initial damage had already occurred to the guidewire.

Breakage or fracture of the guide wire can cause complications including myocardial perforation, pulmonary embolism, arrhythmias, sepsis, endocarditis and even cardiac arrest [12]. The unpredictability of such complications mandates that immediate removal of foreign bodies be addressed. Techniques for removal of foreign bodies vary and depend on the type of fragment, its location, and the experience of the operator [11]. The most commonly used techniques are snare-loop or basket catheter $[11,12]$. In our case, the fractured guidewire entrapped in the CVC was removed using a snare-loop catheter (Fig. 3) without any complications.

We suggest that no force should be applied during insertion or withdrawal of the guidewire if resistance is felt and care should also be exercised when passing the tissue dilator over the guidewire. Furthermore, the snare-loop technique under fluoroscopic guidance could be considered a safe method for the removal of a fractured guidewire.

\section{References}

1. Taylor RW, Palagiri AV. Central venous catheterization. Crit Care 
Med 2007; 35: 1390-6.

2. Seldinger SI. Catheter replacement of the needle in percutaneous arteriography; a new technique. Acta radiol 1953; 39: 368-76.

3. Sternbach G. Sven Ivar Seldinger: catheter introduction on a flexible leader. J Emerg Med 1990; 8: 635-7.

4. Carpentier JP, Braz da Silva J, Choukroun G. Formation of a knot in a J spiral metallic guide: a complication of the Seldinger method. Cah Anesthesiol 1991; 39: 277-8.

5. Shimamoto T, Arai T. Breakage of a Seldinger spring guide wire during percutaneous catheterization of a subclavian vein. Masui 1997; 46: 376-8.

6. Khan KZ, Graham D, Ermenyi A, Pillay WR. Case report: managing a knotted Seldinger wire in the subclavian vein during central venous cannulation. Can J Anaesth 2007; 54: 375-9.

7. Han HS, Jeon YT, Na HS, Hwang JY, Choi EJ, Kim MH. Successful removal of kinked J-guide wire under fluoroscopic guidance during central venous catheterization -A case report-. Korean J Anesthesiol 2011; 60: 362-4.

8. Monaca E, Trojan S, Lynch J, Doehn M, Wappler F. Broken guide wire--a fault of design? Can J Anaesth 2005; 52: 801-4.

9. Wang HE, Sweeney TA. Subclavian central vcenous catheterization complicated by guidewire looping and entrapement. J Emerg Med
1999; 17: 721-4.

10. Garg R, Ramaiah VK, Chouhan RS. Damaged guidewire by the introducer needle tip while inserting central venous catheter in subclavian vein by supraclavicular approach. Saudi J Anaesth 2010; 4: 210-2.

11. Polos PG, Sahn SA. Complication of central venous catheter insertion: fragmentation of a guidewire with pulmonary artery embolism. Crit Care Med 1991; 19: 438-40.

12. Fisher RG, Ferreyro R. Evaluation of current techniques for nonsurgical removal of intravascular iatrogenic foreign bodies. Am J Roentgenol 1978; 130: 541-8.

13. Dagdelen S, Yuce M, Caglar N. Percutaneous removal of two intracardiac and pulmonary truncal catheter fragment by using a snare-loop catheter. Int J Cardiol 2007; 116: 413-5.

14. Merrer J, De Jonghe B, Golliot F, Lefrant JY, Raffy B, Barre E, et al. French Catheter Study Group in Intensive Care. Complications of femoral and subclavian venous catheterization in critically ill patients: a randomized controlled trial. JAMA 2001; 286: 700-7.

15. Mansfield PF, Hohn DC, Fornage BD, Gregurich MA, Ota DM. Complications and failures of subclavian-vein catheterization. $\mathrm{N}$ Engl J Med 1994; 331: 1735-8. 